\title{
Oral intake of heat-killed cells of Lactococcus lactis strain H61 promotes skin health in women
}

\author{
Hiromi Kimoto-Nira*, Reiji Aoki, Keisuke Sasaki, Chise Suzuki and Koko Mizumachi \\ NARO Institute of Livestock and Grassland Science, Ikenodai 2, Tsukuba, Ibaraki 305-0901, Japan
}

(Received 1 February 2012 - Final revision received 21 August 2012 - Accepted 22 August 2012)

Journal of Nutritional Science (2012), vol. 1, e18, page 1 of 7

doi:10.1017/jns.2012.22

\section{Abstract}

We conducted a double-blind, placebo-controlled trial to evaluate the effect of heat-killed cells of Lactococcus lactis strain H61 on various skin properties of Japanese women. Volunteers (age 31-62 years) were randomly assigned to receive test food with or without $60 \mathrm{mg}$ of heat-killed strain H61 (fifteen women in each group; H61 and control groups, respectively) daily for 8 weeks. Results were analysed for three age categories (30s, 40s and 50-60s). Compared with that at week 0 , skin hydration at the inner forearm at weeks 4 and 8 decreased in all volunteers (except those in their 50-60s) because of the environmental change from autumn to winter. The oldest H61 group maintained skin hydration at the inner forearm throughout the study. Skin elasticity and melanin content in the cheek decreased and sebum content increased throughout the test period due to seasonal environmental change, regardless of age or H61 treatment. Self-evaluation scores for apparent hair follicles and dryness of the throat at week 8 were higher in the overall H61 group than in the combined placebo group. The 30s H61 group noted marked improvements in self-surveyed skin elasticity at week 8 compared with at week 0 and with the placebo group at week 8. The results of the present study indicate that oral intake of heat-killed cells of $L$. lactis strain H61 can improve some skin properties and body characteristics in women. This strain would probably be useful in increasing the quality of life in an ageing population.

Key words: Lactic acid bacteria: Skin: Human studies

Many countries are faced with the challenge of maintaining good health and a high quality of life in an ageing population that is living longer than ever before. In this context, the probiotic properties of lactic acid bacteria have garnered particular interest. Probiotics have been defined by the Food and Agriculture Organization-World Health Organization as 'live microorganisms which, when administered in adequate amounts, confer a health benefit to the host'. For example, probiotic lactic acid bacteria can reduce serum lipid content $t^{(1)}$, improve the imbalances of intestinal microbiota ${ }^{(2)}$ and exert immunomodulatory activity ${ }^{(3)}$ in human subjects. Lactic acid bacteria probably have additional probiotic properties as well; our previous study showed that oral administration of heat-killed Lactococcus lactis subsp. cremoris strain H61 to aged senescence-accelerated mice was associated with a decreased incidence of skin ulcers, reduced bone density loss and reduced hair loss compared with those of controls that did not receive strain $\mathrm{H}_{61}{ }^{(4)}$. Strain $\mathrm{H} 61$ has already been shown to be empirically safe through its use in manufactured dairy products in Japan. To fully explore the anti-ageing potential of strain H61, further investigations in human trials are required.

Various endogenous and environmental factors, including ageing, exposure to sunlight, chemicals and mechanical damage ${ }^{(5,6)}$, are known to affect various properties of the skin. In addition, the consumption of various foods is thought to alter the condition of skin. For example, several nutrients including cocoa flavanol ${ }^{(7)}$ and lycopene ${ }^{(8)}$ have been shown to be beneficial in maintaining or improving skin characteristics. In addition, increased intake of vitamin $\mathrm{C}$ and linoleic acid and decreased consumption of fats and carbohydrates are associated with an improved appearance of ageing $\operatorname{skin}^{(9)}$.

In the present study, we investigated the effects of oral supplementation with heat-killed cells of $L$. lactis strain H61 on various properties of human skin. When presented as heatkilled or live cells or in fermented milk, strain H61 improved the condition of the skin of senescence-accelerated mice ${ }^{(4)}$. We used heat-killed cells for this study because they are easier to

* Corresponding author: Dr H. Kimoto-Nira, fax +81 298 388606, email anne@affrc.go.jp 
handle than are viable bacteria. In addition, we analysed the effect of age on the results by dividing the subjects into three age categories (30s, 40s and 50-60s).

\section{Materials and methods}

\section{Subjects and study design}

A total of thirty healthy women (age 31-62 years) from our Institute were enrolled in this study. The study was performed between October 2010 and December 2010 as a randomised, double-blind placebo-controlled application test, in two groups, consisting of fifteen women each. Exclusion criteria were pregnancy, breast-feeding and those who routinely used medical products for various diseases including metabolic, cardiovascular and hepatic diseases. Volunteers were allocated to three age-range categories: $30 \mathrm{~s}$ (35.1 (SE 1.08) years), 40s (44.7 (SE 0.86) years) and 50-60s (54.5 (SE 1.61) years). Age distribution was comparable between the control and treatment populations.

For the preparation of test food including cells of $L$. lactis subsp. cremoris H61, constituted broth $(0.5 \%$ meat extract, $0.5 \%$ yeast extract, $1 \%$ sodium succinate, $1 \%$ sodium chloride and $1 \%$ glucose) was inoculated to the level of $1 \%(\mathrm{v} / \mathrm{v})$ with strain H61 (MAFF 400007) and cultured for $1 \mathrm{~d}$ at $35^{\circ} \mathrm{C}$. Bacterial cells were harvested and washed twice with $0.85 \%$ $\mathrm{NaCl}$ and then resuspended in the same solution. Heat-killed cells were prepared by treatment at $121^{\circ} \mathrm{C}$ for $15 \mathrm{~min}$, followed by centrifugation and lyophilisation. Daily doses totalled $2.0 \mathrm{~g}$ and comprised vehicle (potato starch, lactose and citrate) with or without $60 \mathrm{mg}$ of lyophilised bacterial cells.

The duration of the study was 8 weeks, with assessment of skin parameters at weeks 0,4 and 8 . This study was conducted according to the guidelines in the Declaration of Helsinki, and all procedures involving human subjects were approved by the local medical ethics committee at our Institute. All test subjects received detailed information listing all parameters relevant to the study, and they submitted a written declaration of consent for participation in the study.

\section{Evaluation of skin properties}

Hydration of the skin of the right and left inner forearms was measured, and hydration and other parameters of the right and left lateral angles of the cheeks were assessed in the study volunteers. Before measurements, each subject washed her cheeks once with soap in tap water and sat quietly for 20 min to equilibrate to the conditions in the testing room (22 $\pm 2{ }^{\circ} \mathrm{C}$; relative humidity, $50 \pm 5 \%$ ). Two symmetrical regions of the cheeks were evaluated. Skin hydration was measured by using a corneometer (CM 825, Courage \& Khazaka Electronics $)^{(10,11)}$. Malar skin elasticity was measured by an absorption method (Cutometer SEM575, Courage \& Khazaka Electronics $)^{(11,12)}$. In brief, a 2 -mm region of skin was aspirated at $300 \mathrm{mbar}$ for $2 \mathrm{~s}$ and then released. The ratio of immediate retraction to total distension was recorded. The melanin and sebum contents in the cheeks were measured by using a Mexameter (Courage \& Khazaka Electronics) ${ }^{(13)}$ and a Sebumeter SM810 (Courage \& Khazaka Electronics) ${ }^{(11)}$, respectively. Skin hydration and melanin content were measured three times at each location and time point. Skin elasticity was measured four times at each site and time point. Sebum content was measured once per time point. The skin parameters of each subject were measured before intake and, based on those values, the subjects were randomly assigned to either group.

\section{Self-evaluation}

The self-evaluation questionnaire comprised two sections to assess perceptions of skin-specific attributes (section I) and indictors of general health (section II) at weeks 4 and 8 as compared with week 0 , according to their impressions. Section I addressed skin properties of lustre, elasticity, darkness, spots, sagging, apparent hair follicles, crevices, wrinkles (forehead, mouth and eyes), dryness, stickiness, freedom from blemishes, redness, ability to retain cosmetics, the number of pimples, size of pimples and the presence of dark circles around eyes. Section II included swelling, oversensitivity to cold, paleness of skin, dizziness, stiff neck, headache, energy level, irritation, constipation, diarrhoea, lustre of hair, appetite, dryness of the throat, sweatiness and ease of falling asleep. Subjects evaluated these conditions by responding to questionnaire items by using a five-point scale, in which scores ranged from -2 (the characteristic was markedly worse than previously) to 0 (no change in the characteristic) to +2 (the characteristic was markedly better than previously) for each item.

\section{Statistics}

Data are expressed as least-square means. For all parameters in skin properties and all time points (weeks 0, 4 and 8), descriptive statistics were calculated and were analysed by using a general linear model procedure (SAS version 9.1; SAS Institute). Least-square means were compared by applying the Tukey-Kramer test (PDIFF option of the general linear model procedure). In addition, the interaction between age, treatment and week was tested. Subgroup analyses were performed only when the interaction was significant $(P<0.05)$ or tended towards significance $(0 \cdot 05<P<0 \cdot 1)$. Selfevaluation of skin and body conditions in each subject was analysed by using non-parametric tests (NPAR1WAY procedure of SAS). The effects of treatment, week and age on all parameters were analysed by using the Tukey-Kramer test.

\section{Results}

Mechanical analysis of skin parameters in women after treatment with strain H61

Table 1 indicates the mean measurements associated with various skin parameters in thirty women, as compared according to H61 intake, age and week of analysis. H61 treatment alone had no effect on any skin parameter (Table 1). Age had a significant $(P=0.0162)$ effect only on melanin content in the cheek, which was lower in women in their 30s compared 
Table 1. Effect of heat-killed cells of Lactococcus lactis strain $\mathrm{H} 61$ on skin parameters of women (Least-squares means)

\begin{tabular}{|c|c|c|c|c|c|}
\hline & \multirow{2}{*}{$\begin{array}{l}\text { Inner forearm: } \\
\text { hydration }(A U)\end{array}$} & \multicolumn{4}{|c|}{ Cheek } \\
\hline & & Hydration (AU) & Elasticity & Melanin (MI) & Sebum $\left(\mu \mathrm{g} / \mathrm{cm}^{2}\right)$ \\
\hline \multicolumn{6}{|l|}{ Treatment } \\
\hline Control & 40.5 & 57.5 & 0.317 & 167 & $46 \cdot 2$ \\
\hline $\mathrm{H} 61$ & $42 \cdot 8$ & 59.0 & 0.323 & 173 & $50 \cdot 6$ \\
\hline$P$ & NS & NS & NS & 0.0162 & NS \\
\hline \multicolumn{6}{|l|}{ Age (years) } \\
\hline $30 \mathrm{~s}$ & 38.9 & 59.6 & 0.330 & $141^{\mathrm{b}}$ & 47.5 \\
\hline $40 \mathrm{~s}$ & $41 \cdot 2$ & $56 \cdot 8$ & 0.325 & $188^{\mathrm{a}}$ & 40.5 \\
\hline 50-60s & $44 \cdot 7$ & $58 \cdot 3$ & 0.306 & $181^{a, b}$ & $57 \cdot 1$ \\
\hline$P$ & NS & NS & NS & 0.0162 & NS \\
\hline \multicolumn{6}{|l|}{ Week } \\
\hline 0 & $45 \cdot 1^{a}$ & 57.9 & $0.330^{\mathrm{a}}$ & $188^{a}$ & $15 \cdot 0^{b}$ \\
\hline 4 & $40 \cdot 1^{b}$ & 58.7 & $0.322^{\mathrm{a}}$ & $168^{\mathrm{b}}$ & $54 \cdot 5^{\mathrm{a}}$ \\
\hline 8 & $39 \cdot 6^{b}$ & $58 \cdot 1$ & $0.308^{b}$ & $154^{\mathrm{C}}$ & $75 \cdot 7^{\mathrm{a}}$ \\
\hline$P$ & $<0.0001$ & NS & 0.0014 & $<0.0001$ & $<0.0001$ \\
\hline Treatment $\times$ age: $P$ & NS & NS & NS & NS & NS \\
\hline Treatment $\times$ week: $P$ & NS & NS & NS & NS & NS \\
\hline Age $\times$ week: $P$ & NS & NS & 0.0149 & NS & NS \\
\hline Treatment $\times$ week $\times$ age: $P$ & 0.0851 & NS & NS & NS & NS \\
\hline
\end{tabular}

$\mathrm{AU}$, arbitrary units; MI, melanin index.

a,b,c Mean values within a category (treatment, age and week) within a column with unlike superscript letters were significantly different $(P<0.05)$

with the other two age groups (Table 1). Week (seasonal change) had a significant $(P<0 \cdot 001)$ effect on all measured parameters except cheek hydration (Table 1). Skin hydration of the inner forearm $(P<0 \cdot 0001)$, cheek elasticity $(P=$ $0 \cdot 0014)$ and melanin content $(P<0 \cdot 0001)$ decreased, whereas sebum content in cheeks $(P<0.0001)$ increased in response to environmental changes, such as temperature and UV exposure, from autumn to winter.

Neither interaction between treatment and age nor interaction between treatment and week had an effect on any parameters of the inner forearm or cheek. A significant $(P=$ $0 \cdot 0149)$ interaction between age and week was obtained for cheek elasticity, in that women in their 30s had higher elasticity at week 0 than at week 8 (Fig. 1). Skin elasticity in cheeks was significantly $(P<0 \cdot 05)$ higher in the youngest group than in the oldest women.

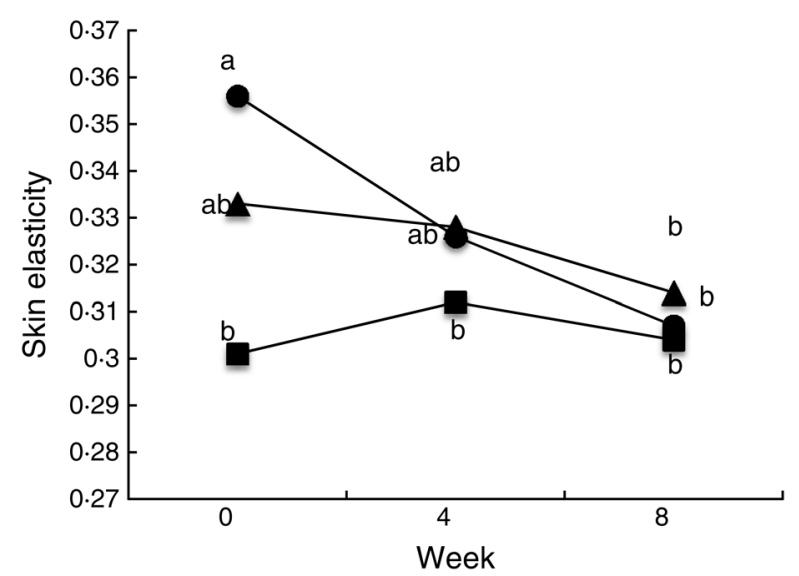

Fig. 1. Skin elasticity of cheeks of women in three age categories $(30 \mathrm{~s},-$ 40s, - - - - 50-60s, - $\mathbf{-}$-) who received a placebo or heat-killed cells of Lactococcus lactis strain H61. Values are means (eight to twelve subjects). a,b Values with unlike letters were significantly different $(P<0.05)$.
Interaction between treatment, week and age category on hydration of the inner forearm tended towards significance $(P=0.0851$; Fig. 2). Skin hydration in the inner forearm was significantly decreased in all subjects in their 30 s in the H61 group and 40s in the placebo and H61 groups at week 4 ( $P$ $<0.05)$ or week $8(P<0.05)$ compared with week 0 . In the subjects in their 30s who received the placebo, skin hydration in the inner forearm at weeks 4 and 8 also decreased compared with week 0 , but these differences were not significant. In contrast, dehydration in 50-60-year-old subjects given strain H61

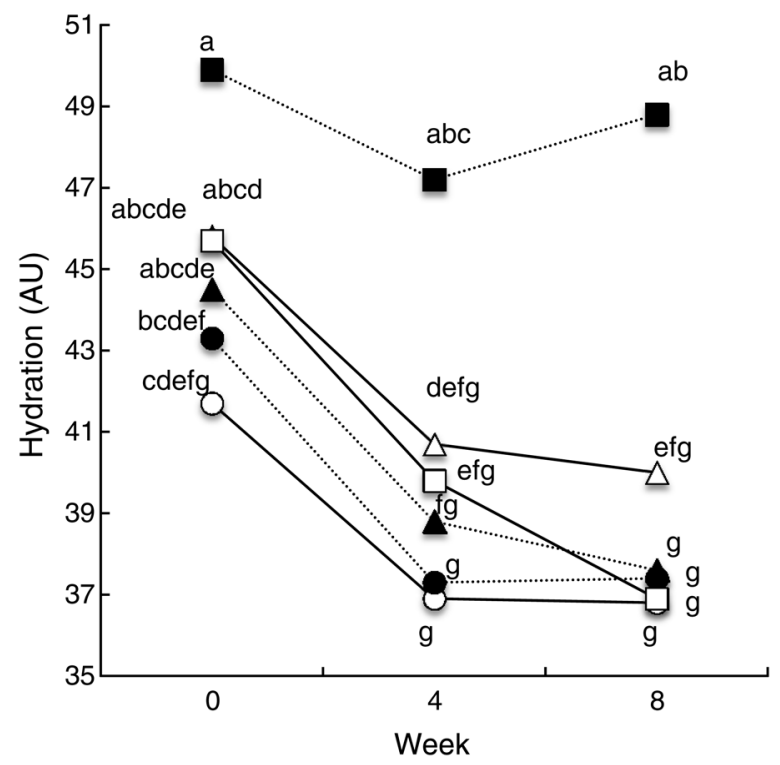

Fig. 2. Skin hydration of the inner forearm of women in three age categories (30s, 40s and 50-60s) who received a placebo or heat-killed cells of Lactococcus lactis strain H61. -O-, Placebo (30s); .. -.. H61 (30s); - $\Delta-$, placebo (40s); ..ム.., H61 (40s); - $\square-$, placebo (50-60s); .. $\square . ., ~ H 61$ (50-60s); $\mathrm{AU}$, arbitrary units. Values are means (four to six subjects). ${ }^{\mathrm{a}-\mathrm{g}}$ Values with unlike letters were significantly different $(P<0.05)$. 
Table 2. Effect of heat-killed cells of Lactococcus lactis strain $\mathrm{H} 61$ on responses to a self-evaluation questionnaire regarding skin and body condition in women (Least-squares means)

\begin{tabular}{|c|c|c|c|c|c|c|}
\hline & \multicolumn{3}{|c|}{ Skin condition } & \multicolumn{3}{|c|}{ Body condition } \\
\hline & Elasticity & Lustre & Apparent hair follicles & Swelling & Dizziness & Dryness of the throat \\
\hline \multicolumn{7}{|l|}{ Treatment } \\
\hline Control & 0.056 & 0.028 & 0.097 & 0.067 & -0.006 & 0.028 \\
\hline $\mathrm{H} 61$ & 0.080 & 0.225 & 0.219 & 0.111 & 0.050 & 0.160 \\
\hline$P$ & NS & NS & NS & NS & NS & NS \\
\hline \multicolumn{7}{|l|}{ Age (years) } \\
\hline $30 \mathrm{~s}$ & 0.150 & 0.150 & 0.100 & 0.100 & -0.100 & 0.100 \\
\hline $40 \mathrm{~s}$ & 0.054 & 0.125 & 0.125 & 0.042 & 0.042 & 0.113 \\
\hline $50-60 s$ & 0.000 & 0.375 & 0.250 & 0.125 & 0.125 & 0.063 \\
\hline$P$ & NS & NS & NS & NS & 0.0474 & NS \\
\hline \multicolumn{7}{|l|}{ Week } \\
\hline 4 & 0.037 & $0 \cdot 111^{\mathrm{b}}$ & 0.172 & 0.067 & -0.025 & 0.052 \\
\hline 8 & 0.098 & $0.322^{\mathrm{a}}$ & 0.144 & 0.111 & 0.069 & 0.132 \\
\hline$P$ & NS & 0.0066 & NS & NS & NS & NS \\
\hline Treatment $\times$ age: $P$ & 0.0984 & NS & NS & 0.0332 & NS & NS \\
\hline Treatment $\times$ week: $P$ & 0.0086 & NS & 0.0302 & 0.0125 & NS & 0.0410 \\
\hline Age $\times$ week: $P$ & NS & NS & NS & 0.0380 & NS & NS \\
\hline Treatment $\times$ week $\times$ age: $P$ & 0.0139 & 0.0753 & NS & NS & NS & NS \\
\hline
\end{tabular}

${ }^{\mathrm{a}, \mathrm{b}}$ Mean values within a category (treatment, age and week) within a column with unlike superscript letters were significantly different $(P<0.05)$.

cells was suppressed $(P<0 \cdot 05)$ at weeks 4 and 8 of treatment compared with week 0 .

\section{Self-evaluation of skin and body conditions after treatment with strain $\mathrm{H} 61$}

Table 2 indicates the self-scored skin (elasticity, lustre and apparent hair follicles) and body (swelling, dizziness and dryness of the throat) conditions that yielded significant results concerning the effects of H61 treatment. H61 treatment had improvement effects on all of the items in Table 2, although they were not significant. Improvement of dizziness was significantly $(P=0.0474)$ affected by age, but a significant difference was not observed in age distribution after analysis using the Tukey-Kramer test. Week had a significant $(P=0.0066)$ effect only on the skin parameter of lustre, which was higher at week 8 than week 4 .

Treatment and age tended to interact in the elasticity of the cheek $(P=0.0984)$ and significantly $(P=0.0332)$ interacted in swelling, but Tukey-Kramer analysis failed to reveal any significant difference in these results.

Elasticity of the cheek $(P=0.0086)$, apparent hair follicles in the cheek $(P=0.0302)$, swelling $(P=0.0125)$ and dryness of the throat $(P=0.0410)$ all showed significant interaction of treatment and week (Table 2). At week 8 , women who received H61 treatment reported significant $(P<0 \cdot 05)$ improvement regarding apparent hair follicles in the cheek (Fig. 3(A)) and dryness of the throat (Fig. 3(B)). TukeyKramer analysis failed to identify significant differences regarding elasticity in the cheek and swelling in subjects.

Elasticity $(P=0 \cdot 0139$; Fig. 4) showed significant interaction between treatment, week and age. Women in the 30s group who received H61 treatment reported a significant $(P<0 \cdot 05)$ improvement in elasticity at week 8 compared with week 0 and with the placebo group at week 8 . Lustre $(P=0.0753)$ also showed a trend toward significance between treatment, week and age, but Tukey-Kramer analysis failed to confirm a significant difference in these results.
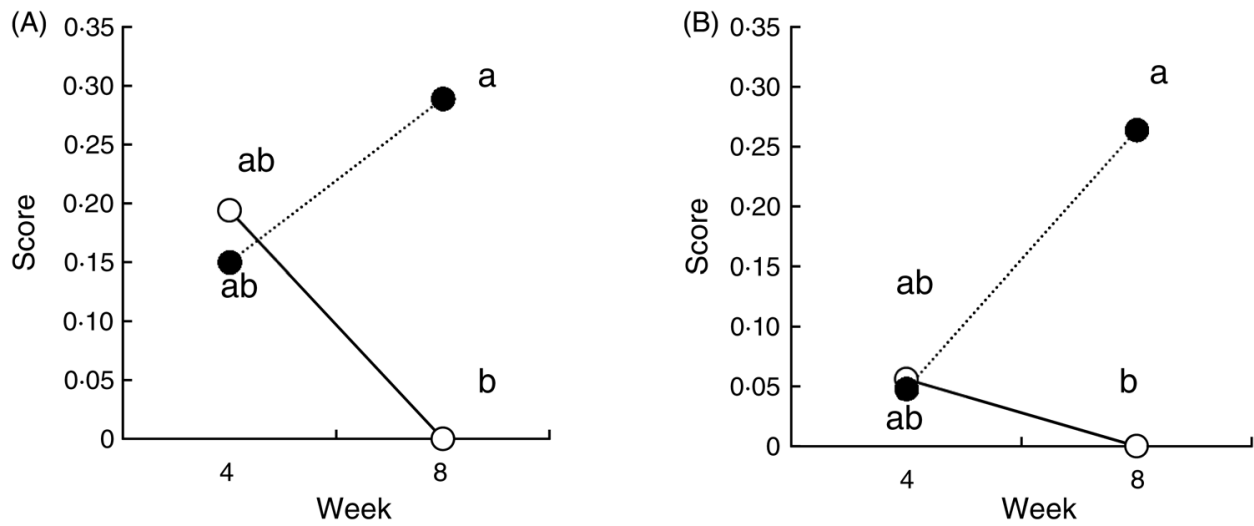

Fig. 3. Self-evaluation of (A) apparent hair follicles and (B) dryness of the throat (score, -2 to +2 ; relative to week 0 ) in women (31-62 years) who received a placebo (-O-) or heat-killed cells of Lactococcus lactis strain H61 (..- ..). Values are means (fifteen subjects). ${ }^{\text {a,b }}$ Mean values with unlike letters were significantly different $(P<0.05)$. 


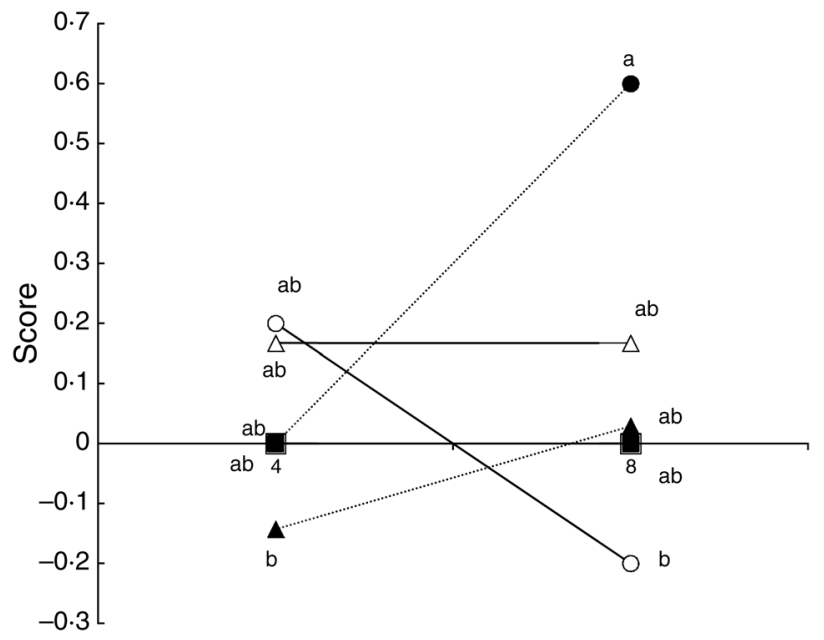

Week

Fig. 4. Self-evaluation of elasticity (score, -2 to +2 ; relative to week 0 ) of the cheeks of women in three age categories (30s, 40 s and $50-60$ s) who received a placebo

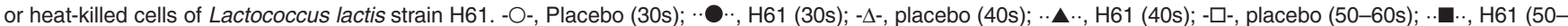
60s). Values are means (four to six subjects). ${ }^{\text {a,b }}$ Values with unlike letters were significantly different $(P<0.05)$

\section{Discussion}

The present study represents an attempt to modulate the skin parameters of women through the intake of heat-killed cells of L. lactis strain H61 and to clarify the effect of this treatment on the skin of women in various age cohorts. Oral intake of 40 $\mathrm{mg}$ of soya isoflavone aglycones daily has been reported to improve age-associated attributes of skin (e.g. fine wrinkles and malar skin elasticity) of middle-aged (from late 30 s to early 40s) Japanese women ${ }^{(12)}$. Skin elasticity and the degree of dryness in the cheeks of Japanese women (age 20-39 years) were improved after 4 weeks of consuming yogurt prepared by using Lactobacillus delbrueckii subsp. bulgaricus 2038 and Streptococcus thermophilus $1131^{(14)}$. However, no previous reports have addressed the effect of heat-killed lactic acid bacterial cells on human skin properties and age distribution. Here, we show that oral intake of heat-killed strain H61 cells was associated with suppression of dehydration due to seasonal change in the inner forearms of women in their 50-60s but not in their 30 s and 40 s. In response to a self-evaluation questionnaire, subjects in their 30 s reported significant improvements in elasticity after treatment with H61 cells, compared with that at week 0 , although the results were inconsistent with the data from the mechanical analysis. It is notable that the effect of strain H61 on some skin properties varied according to the age category (30s, 40s and 50-60s). However, the reason for this variation remains unclear as yet.

By what mechanism, then, does strain H61 specifically improve the properties of ageing skin in women? According to responses to the self-evaluation questionnaire, H61 treatment had no effect on general-health items such as constipation, diarrhoea and sleep patterns, which reportedly are associated with skin health ${ }^{(14)}$. Reactive oxygen species, including singlet oxygen and superoxide, are induced in the skin by solar UV radiation and have long been suspected of contributing to the deleterious effects of cutaneous photodamage ${ }^{(15)}$. Reactive oxygen species promote lipid peroxidation, protein oxidation and cross-linking, enzyme inactivation, and DNA damage ${ }^{(15)}$. Studies using human dermal fibroblasts have suggested that singlet oxygen mediates UVA (320-400 $\mathrm{nm}$ )-induced up-regulation of interstitial collagenase in the skin, through the induction of the inflammatory cytokines IL-1 and IL-6 ${ }^{(16)}$. Oral administration of antioxidant substances such as vitamin $C^{(7,17)}$ and carotenoids ${ }^{(18)}$ has been shown to have photoprotective effects on the skin. In our preliminary study, heat-killed cells of strain H61 had an antioxidant effect by scavenging the $\alpha, \alpha$-diphenyl- $\beta$-picrylhydrazyl radical. We are currently investigating potential additional antioxidative effects of heat-killed strain H61, including suppression of oxidative damage of DNA and reactive oxygen species generation in macrophage cells.

In addition, immune responses are closely associated with skin properties. Exposure to UVB (280-320 nm) can impair specific and non-specific immune responses ${ }^{(19)}$, and UVB-induced immunomodulation contributes to photocarcinogenesis ${ }^{(20)}$. In mice, UV exposure reduced the production of T helper type 1 cytokines including IL- 2 and interferon- $\gamma$ and increased the synthesis of the $\mathrm{T}$ helper type 2 cytokines IL-4, IL-5 and IL-10 $0^{(21)}$. In our previous study ${ }^{(4)}$, spleen cells from mice fed with strain H61 produced more interferon- $\gamma$ and IL-12 than did those from control mice that did not receive strain H61, suggesting that administration of strain H61 enhanced T helper type 1 immune responses. Therefore, the immunomodulatory activity of strain H61 in enhancing $\mathrm{T}$ helper type 1 responses might underlie its ability to improve various properties of ageing skin. Further investigation is needed to investigate oxidative status and immune responses by analysing serum from subjects supplemented with the cells of strain H61, in order to clarify the mechanism of promoting skin health and the differences in the effect of strain H61 among age categories.

Aged skin has several typical characteristics, including fine wrinkles, sallowness and loss of elasticity ${ }^{(22)}$. In addition, it has been reported that the secretion of sebum in women reaches a peak during their $20 \mathrm{~s}$ and declines thereafter ${ }^{(23)}$. Melanin 
provides varying degrees of brown coloration at the skin surface, and melanin content is affected by internal and external factors such as age, race and sunlight exposure. Our present data showed that skin hydration, elasticity and sebum content did not differ among age categories, but melanin content in the cheek varied with age. With regard to skin hydration, although skin in the elderly is generally thought to be dry ${ }^{(24,25)}$, adult women (age 30-48 years) showed higher skin hydration in the cheek and inner forearm than did their children (age 10-14 years) ${ }^{(26)}$. In another study ${ }^{(27)}$, hydration of normal skin did not differ between young and elderly women. Therefore, data regarding the effect of age on skin hydration are contradictory. The use of instruments that are based on different measurement principles and that assess the skin at different depths and subjects with different ethnic backgrounds may account for the differences reported in the literature.

Seasonal effect is an important factor contributing to skin condition. Skin disorders such as psoriasis and senile xerosis tend to exacerbate in winter. In Japanese women, the hydration state of the stratum corneum on the flexor forearm was significantly lower during winter than during summer ${ }^{(28)}$. Our present study showed similar seasonal changes in skin properties: hydration in the forearm and elasticity and melanin content in the cheek were lower during winter than during autumn, whereas sebum content in the cheek was higher in winter. Thermal conditions are apt to affect body temperature regulation and water metabolism in the elderly ${ }^{(29)}$, and one's sensitivity to changes in air temperature declines with age ${ }^{(30)}$. In the present study, changes in environmental factors such as temperature and humidity from autumn to winter affected skin properties in our subjects. Furthermore, one study ${ }^{(28)}$ reported that exposed facial skin became more irritated under the influence of the dry and cold environment of winter than did the forearm. However, the present study showed that seasonal changes were apparent in the hydration of the inner forearm but not the cheek. This difference in hydration may be one of the reasons underlying the apparent effect of strain H61 in the inner forearm in this study. Repeating the examinations used in the present study but during a different season may help to clarify this issue.

In conclusion, we used here a non-invasive mechanical method and self-evaluation survey to show that heat-killed cells of $L$. lactis strain H61 improved skin properties such as skin hydration and apparent fair follicles in middle-aged and aged Japanese women. Although the subjects' impressions should be given much weight, these impressions regarding the effects of oral intake of strain H61 on skin properties (e.g. apparent hair follicles) should be confirmed in future studies by using appropriate measurable instruments.

\section{Acknowledgements}

The present study was supported in part by a grant for the project 'Development of evaluation and management methods for supply of safe, reliable, and functional food and farm procedure' from the Japanese Ministry of Agriculture, Forestry, and Fisheries. We thank all the volunteers who participated in this study. H. K.-N. initiated and organised the study and wrote the article. R. A. provided technical assistance. K. S. performed the statistical analyses and helped to plan the experimental design. C. S. and K. M. were involved in the conception of the study design and gave final approval of the manuscript. None of the authors had a personal or financial conflict of interest.

\section{References}

1. Higashikawa F, Noda M, Awaya T, et al. (2010) Improvement of constipation and liver function by plant-derived lactic acid bacteria: a double-blind, randomized trial. Nutrition 26, 367-374.

2. Lidbeck A, Geltner-Allinger U, Orrhage KM, et al. (1991) Impact of Lactobacillus acidophilus supplements on the fecal microflora and soluble faecal bile acids in colon cancer patients. Microbiol Ecol Health Dis 4, 81-88.

3. Kalliomaki M, Salminen S, Poussa T, et al. (2003) Probiotics and prevention of atopic disease: 4-year follow-up of a randomised placebo-controlled trial. Lancet 361, 1869-1871.

4. Kimoto-Nira H, Suzuki C, Kobayashi M, et al. (2007) Anti-aging effect of a lactococcal strain: analysis using senescence-accelerated mice. Br J Nutr 98, 1178-1186.

5. Pathank MA (1982) Sunscreens: tropical and systemic approaches for protection of human skin against harmful effects of solar radiation. J Am Acad Dermatol 7, 285-312.

6. McCallion R \& Li Wan Po A (1993) Dry and photo-aged skin: manifestations and management. J Clin Pharm Ther 18, 15-32.

7. Heinrich U, Neukam K, Tronnier H, et al. (2006) Long-term ingestion of high flavonol cocoa provides photoprotection against UV-induced erythema and improves skin condition in women. J Nutr 136, 1565-1569.

8. Stahl W, Heinrich U, Aust O, et al. (2006) Lycopene-rich products and dietary photoprotection. Photochem Photobiol Sci 5, 238-242.

9. Cosgrove MC, Franco OH, Stewart PG, et al. (2007) Dietary nutrients intakes and skin-aging appearance among middle-aged American women. Am J Clin Nutr 86, 1225-1231.

10. De Spirt S, Stahl W, Tronnier H, et al. (2009) Intervention with flaxseed and borage oil supplements modulates skin condition in women. Br J Nutr 101, 440-445.

11. Nagata C, Nakamura K, Wada K, et al. (2010) Association of dietary fat, vegetables and antioxidant micronutrients with skin ageing in Japanese women. Br J Nutr 103, 1493-1498.

12. Izumi T, Saito M, Obata A, et al. (2007) Oral intake of soy isoflavone aglycone improves the aged skin of adult women. J Nutr Sci Vitaminol 53, 57-62.

13. Huh SY, Shin JW, Huh CH, et al. (2010) The efficiency and safety of 4-n-butylrescorcinol $0 \cdot 1 \%$ cream for the treatment of melasma: a randomized controlled split-face trial. Ann Dermatol 22, 21-25.

14. Isawa K, Noma T, Yamamoto M, et al. (2008) Verifying the ability of yogurt prepared with LB81 lactic acid bacteria to improve skin function (article in Japanese). $J$ Int Microbiol 22, 1-5.

15. Carbonare MD \& Pathak MA (1992) Skin photosensitizing agents and the role of reactive oxygen species in photoaging. $J$ Photochem Photobiol 14, 105-124.

16. Wlaschek M, Wenk J, Brenneisen J, et al. (1997) Singlet oxygen is an early intermediate in cytokine-dependent ultraviolet-A induction of interstitial collagenase in human dermal fibroblasts in vitro. FEBS Lett 413, 239-242.

17. Darr D, Comb S, Dunston S, et al. (1992) Topical vitamin C protects procine skin from ultraviolet radiation-induced damage. $\mathrm{Br} \mathrm{J}$ Dermatol 127, 247-253.

18. Alaluf S, Heinrich U, Stahl W, et al. (2002) Dietary carotenoids contribute to normal human skin color and UV photosensitivity. J Nutr 132, 399-403.

19. Hurks M, Garssen J, van Loveren H, et al. (1994) General aspects of UV-irradiation on the immune system. In Photobiology in Medicine, p. 161 [G Jori, editor]. New York: Plenum Press. 
20. Kripke ML (1989) Immunologic mechanisms in UV-radiation carcinogenesis. Adv Cancer Res 34, 69-106.

21. Araneo BA, Dowel T, Moon HB, et al. (1989) Regulation of murine lymphokine production in vivo. Ultraviolet radiation exposure depresses IL-2 and enhances IL-4 production by T cells through an IL-1 dependent mechanism. J Immunol 146, 1737-1744.

22. Makrantonaki E \& Zouboulis CC (2007) Characteristics and pathomechanisms of endogenously aged skin. Dermatology 214, 352-360.

23. Yamamoto A (1991) Skin surface lipids (article in Japanese). J Cos Sci Soc 15, 247-249.

24. Potts RO, Buras EM \& Chrisman DA (1984) Changes with age in the moisture content of human skin. J Invest Dermatol 82, 97-100.

25. Rogiers V, Derde MP, Verleye G, et al. (1990) Standardized conditions needed for skin surface hydration measurements. Cosmet Toilet $105,73-82$.
26. Akutsu N, Ooguri M, Onodera T, et al. (2009) Functional characteristics of the skin surface of children approaching puberty: age and seasonal influences. Acta Derm Venereol 89, 21-27.

27. Frodin T, Helander P, Molin L, et al. (1988) Hydration of human stratum corneum studied in vivo by optothermal infrared spectrometry, electrical capacitance measurement, and evaporimetry. Acta Derm Venereol 68, 461-467.

28. Kikuchi K, Kobayashi H, Fur IL, et al. (2002) The winter season affects more severely the facial skin than the forearm skin: comparative biophysical studies conducted in the same Japanese females in later summer and winter. Exog Dermatol 1, 32-38.

29. Tochihara Y, Ohnaka T, Nagai Y, et al. (1993) Physiological responses and thermal sensations of the elderly in cold and hot environments. $J$ Therm Biol 5/6, 335-361.

30. Collins KJ, Exton Smith AN \& Dore C (1981) Urban hypothermia: preferred temperature and thermal perception in old age. Br Med J 282, 175-177. 